\title{
HMM BASED FAST KEYWORD SPOTTING ALGORITHM WITH NO GARBAGE MODELS
}

\author{
S. Sunil, Supriyo Pa!it and T.V. Sreenivas \\ CEDT, Indian Institute of Science, Bangalore 560012, India. \\ E-mail : ssunil@cedt.iisc.ernet.in
}

\begin{abstract}
The problem of discriminating keyword and non-keyword speech which is important in wordspotting applications is addressed here. We have shown that garbage models cannot reduce both rejection and false alarm rates simultaneously. To achieve this we have proposed a new scoring and search method for HMM based wordspotting without garbage models. This is a simple forward search method which incorporates the duration modelling of keyword for efficient discrimination of keyword and non-keyword speech. This method is computationally fast, which makes it suitable for real-time implementation. The results are reported on a speaker independent database containing 10 keywords embedded in 150 carrier sentences.
\end{abstract}

\section{Introduction}

Keyword spotting methods (KWS) using hidden Markov models (HMM) are based on continuous speech recognition (CSR) algorithms [1,2] where HMMs are used to model both keyword speech and non-keyword speech (comprising of background speech and non-speech sounds). The performance of these methods rely heavily on the performance of nonkeyword speech models also known as "garbage" models. It has been shown $[1,2]$ that by explicitly training garbage HMMs to model the entire background environment, the performance of the keyword spotting system can be improved. In most cases, garbage and keyword HMMs are connected as a network of HMMs and frame synchronous network (FSN) search method [3] is used to decode the test utterance into a sequence of garbage and keyword models. This approach is computationally complex and would be difficult for real-time implementation. The main difference between KWS algorithms is the way in which garbage models are defined and scoring methods are formulated.

\section{Drawbacks of garbage modelling}

The main aim of reducing the rejection rate and false alarm rate simultaneously cannot be achieved with garbage models. For a keyword to be recognised when it occurs in an utterance, we require

$$
P\left(\mathbf{O}^{i} \mid \lambda_{i}\right)>P\left(\mathbf{O}^{i} \mid \lambda_{g}\right) \quad 1 \leq i \leq M
$$

where $\lambda_{i}=\mathrm{HMM}$ of keyword $-i, \mathbf{O}^{i}=$ utterance of keyword $-i, \lambda_{g}=$ garbage HMM and $\mathrm{M}=$ number of keywords in the vocabulary. To prevent false alarms, we need

$$
P\left(\mathbf{O}^{g} \mid \lambda_{g}\right)>P\left(\mathbf{O}^{g} \mid \lambda_{i}\right) \quad 1 \leq i \leq M
$$

where, $\mathbf{O}^{s}=$ garbage utterance. Since the non-keyword speech set is very large compared to the keyword set, the score for keyword and garbage utterances for garbage model will be comparable, i.e.,

$$
P\left(\mathbf{O}^{i} \mid \lambda_{g}\right) \approx P\left(\mathbf{O}^{s} \mid \lambda_{g}\right) \quad 1 \leq i \leq M
$$

Thus, the following relation becomes apparent from the above inequalities

$$
P\left(\mathbf{O}^{i} \mid \lambda_{i}\right)>P\left(\mathbf{O}^{i} \mid \lambda_{\boldsymbol{g}}\right) \approx P\left(\mathbf{O}^{g} \mid \lambda_{\boldsymbol{g}}\right)>P\left(\mathbf{O}^{\boldsymbol{s}} \mid \lambda_{i}\right)
$$

The first inequality determines the number of rejections and the second determines the number of false alarms. The $\mathrm{ML}$ training of $\lambda_{i}$ and $\lambda_{g}[1,2]$ does not always assure the above inequalities. Even, the on-line garbage modelling technique [4] could achieve low rejection rates at the expense of increased false-alarms. Inequality (4) also shows that garbage models cannot reduce rejection and false-alarm rates simultaneously, because, if we try to reduce $P\left(\mathbf{O}^{i} \mid \lambda_{g}\right)$ to achieve a lower rejection rate (inequality 1 ), this would also reduce $P\left(\mathbf{O}^{g} \mid \lambda_{g}\right)$ (relalion 3) which would increase the false-alarms (inequality 2) and vice-versa. Thus both the rejection and false-alarm rates can be improved only by increasing the separation between $P\left(\mathbf{O}^{i} \mid \lambda_{i}\right)$ and $P\left(\mathbf{O}^{g} \mid \lambda,\right)$ which shows that garbage models are not crucial for improving the performance of keyword spotters. Based on these arguments we propose a new technique of keyword spotting without garbage models.

\section{No garbage model technique}

Let $K_{i}, 1 \leq i \leq M$ be the set of $\mathrm{M}$ distinct keywords and let $\lambda_{K}$ be the corresponding keyword models. We denote the observation sequence corresponding to the utterance as $O\left(=O_{1}, O_{2}, \ldots, O_{T}\right), T$ being the length of the utterance. Let $S_{i}\left(t_{1}, t_{2}\right)$ be the score for a keyword $-i, 1 \leq i \leq M$, to occur in the observation interval from $t_{1}$ to $t_{2}$, which is formulated as

$$
\begin{aligned}
& S_{i}\left(t_{1}, t_{2}\right)= \\
& \frac{\log P_{4}\left(q_{t_{1}}^{*} \cdots q_{t_{2}}^{*}, O_{t_{1}}, \cdots_{\nu} O_{t_{2}} \mid \lambda_{i}\right)+\log P_{d}\left(t_{2}-t_{1} \mid D_{i}\right)}{t_{2}-t_{1}}
\end{aligned}
$$

where $P_{v}=$ Viterbi likelihood score, $P_{d}=$ Duration probability for the keyword model $D_{i}$.

For the observation sequence $O_{1}, \cdots, O_{T}$,

$$
\left[i^{*}, t_{1}^{*}, t_{2}^{*}\right]=\arg \max S_{i, t_{1}, t_{2}}\left(t_{1}, t_{2}\right)
$$

is determined for $1 \leq i \leq M, 1 \leq t_{1} \leq T, 1 \leq t_{2} \leq T$ such that $d_{i}^{\min } \leq\left(t_{2}-t_{1}\right) \leq d_{i}^{\max }$ where $d_{i}^{\min }$ and $d_{i}^{\max }$ are the limits of the duration of keyword - $i$, estimated apriori. The 
keyword $i^{\bullet}$ which maximizes $S_{i}\left(t_{1}, t_{2}\right)$ is the recognised keyword and $t_{1}^{*}$ and $t_{2}^{*}$ are the starting and ending locations.

\subsection{Time Normalization}

The $\log$ Viterbi probability,

$\log P_{v}\left(q_{i_{1}}^{*}, \cdots q_{i_{1}}^{*}, O_{t_{1}}, \cdots, O_{t_{2}} \mid \lambda_{i}\right)$ has a large value for short observation lengths and decreases monotonically for large observation lengths as shown in Figure 1. The log Viterbi probability being negative, this bias can be reduced by dividing it by the length of the observation sequence, $\left(t_{2}-t_{1}\right)$. But experiments showed that, although time normalization works well for large observation lengths (i.e., remains more or less unbiased), it is unable to unbias properly the $\log$ Viterbi probability for very short sequences. This is because, for very short length sequences the log Viterbi probability is so high that even duration normalization is ineffective.

\subsection{Duration Modelling}

To reduce the bias of the log Viterbi probability for short observation lengths a word duration penalty is introduced. The mean $m_{i}$ and variance $\sigma_{i}^{2}, 1 \leq i \leq M$, of the duration of each keyword $K_{i}, 1 \leq i \leq M$ are obtained from the training data. We fit a probability distribution , $D_{i}$. using the mean and variance as parameters. Gamma distribution is used as the probability density function, which has a range from $d=0$ to $\infty, d$ being the duration random variable. The Gamma density falls off quite sharply to 0 as $d$ decreases to 0 , whereas it goes asymptotically to 0 as $d$ tends to $\infty$. The word duration penalty, $\log P_{d}\left(t_{2}-t_{1} \mid D_{i}\right)$ in equation (5) removes the bias of the log Viterbi probability for short length sequences without affecting the higher length sequences significantly. This can be seen from Figure 2 which plots the time normalized log Viterbi probability (a) and the score $S_{i}\left(t_{1}, t_{2}\right)$ (b) for the same values of $t_{1}(=43)$ and $t_{2}$ and for the same keyword model and for the same keyword utterance. From the figure 2(a), for the log Viterbi probability with time normalization there is a local maxima for $t_{2}=73$ (which is the correct ending point) but, this maxima is overshadowed by a maxima for a much short duration, for $\mathrm{t}_{2}=56$ (duration being 14 observation symbols). It can be seen from the figure 2(b) that the score, $S_{i}\left(t_{1}, t_{2}\right)$ suppressed the spurious short duration peak and the maxima at $t_{2}=73$ is kept unaltered. Thus the word duration penalty, $\log P_{d}\left(t_{2}-t_{1} \mid D_{i}\right)$ promotes more probable durations and penalizes the less probable ones.

\subsection{Algorithm}

The complete algorithm is described below,

1. Get the observation sequence $O_{1}, \ldots O_{T}$ for a particular speech utterance.

2. Start with $i=1, i$ being the keyword index

3. $t_{1}=1$

4. If $t_{1}+d^{i}{ }_{\text {min }}-1>T$, go to step 8 .
5. Calculate $S_{i}\left(t_{1}, t_{2}\right)$ from the observation sequence $O_{i} \ldots O_{t_{i}}$ and $\lambda_{i}$ where

$t_{1}-d^{\prime}{ }_{\text {min }}-1 \leq t_{2} \leq \min \left[T, t_{1}+d^{\prime}{ }_{\max }-1\right]$

6. If $S_{i}\left(t_{1}, t_{2}\right)$ is maximum over all the keyword scores considered so far, store keyword index $i$ and the time instants $t_{1}$ and $t_{2}$.

7. $t_{1}=t_{1}+1$, go to step 3

8. $i=i+1$, go to step 3 for $2 \leq i \leq M$, $\mathrm{M}$ being the number of keywords.

After termination, the keyword which gave the maximum score, $S_{i} \cdot\left(t_{1}^{*}, i_{2}^{*}\right), 1 \leq i^{*} \leq M$ its starting $\left(t_{1}^{*}\right)$ and ending locations $\left(i_{2}^{*}\right)$ are obtained. Threshold score $\left(S_{i}^{\prime}\right) \quad 1 \leq i \leq M$, for each keyword is estimated apriori using Baye's Minimum Error Criterion. If $S_{i}\left(t_{1}^{*}, t_{2}^{*}\right) \geq\left(S_{i}^{\prime}\right)$, then the recognized keyword is $i^{*}$, else no keyword is present.

The above algorithm can be visualized as searching for a keyword within a window of observation sequence for each keyword model. The window is slided across the entire observation sequence. This is shown in Figure 3. The size of the window is determined by $d_{i}^{\min }$ and $d_{i}^{\max }, 1 \leq i \leq M$. The search limits $d_{i}{ }^{\min }$ and $d_{i}^{\max }, 1 \leq i \leq M$ are set at $d_{\min }^{i}=m_{i}-0.5 \sigma_{i}$ and $d_{\max }^{i}=m_{i}+3 \sigma_{i}$.

By restricting the search within $d_{i}^{\min }$ and $d_{i}^{\max }$ we eliminate all garbage sequences outside this range from causing false alarms. For sequences within the allowed limit, the contrast between keyword and garbage is achieved by the Viterbi probability and the apriori duration weightage incorporated in the score. Thus, this method improves the separation between $P\left(\mathbf{O}^{i} \mid \lambda_{i}\right)$ and $P\left(\mathbf{O}^{g} \mid \lambda_{i}\right)$

\section{Experiment}

Experiments are conducted on a ten keyword database ( Indian city names, with at most one keyword per sentence ), with each keyword modelled with a 5 state LR discrete HMM. Feature vector includes 10 weighted cepstral coefficients, energy, ratio of residual energy and zeroeth autocorrelation coefficient, zero crossing rate and delta energy. Vector quantization is done using a 256 size codebook designed using LBG algorithm. The recognition results and duration statistics are shown in Table -1 and Table -2 respectively. It is found from the experiments that the insertions usually have shorter durations than the expected keyword durations. It can be seen from Table - 3 that by restricting the lower search limit, $d_{i}^{\min }$, keeping the upper limit $d_{i}^{\max }$ and thresholds fixed, the false alarms have reduced significantly. Figure 4 gives the system performance for two values of $d_{i}{ }^{\text {min }}$ as a function of threshold.

\section{Conclusion}

The major contribution of this paper has been to show that good keyword spotting performance can be obtained from an HMM system using no-garbage model. From the results we can see that the no-garbage model approach shows reasonably good recognition accuracy at a low false alarm rate. These 
results can be compared with that of Rose [2], who has used very elaborate modelling of non-keyword speech. This technique is also computationally fast which makes it possible to do word-spotting in real time. The performance can be further improved by using discriminate techniques of keyword training for increasing the separation between $P\left(\mathbf{O}^{i} \mid \lambda_{i}\right)$ and $P\left(\mathbf{O}^{s} \mid \lambda_{i}\right)$.

\section{References}

[1] Wilson et. al., (1990). Automatic recognition of keywords in unconstrained speech using hidden Markov models. IEEE

Trans. on ASSP 38, 1870-1878.
[2] R. C. Rose (1995). Keyword detection in conversational speech utterances using hidden Markov model based continuous speech recognition. Comp. Speech and Lang. 9, 309-333.

[3] Lee et. al., (1989). A frame synchronous network search algorithm for connected word recognition. IEEE Trans. on ASSP 37, 1649-1658

[4] Bourlard (1994). Oprimizing recognition and rejection performance in word spotting systems. Proc. of ICASSP, Vol. $1,373-376$.

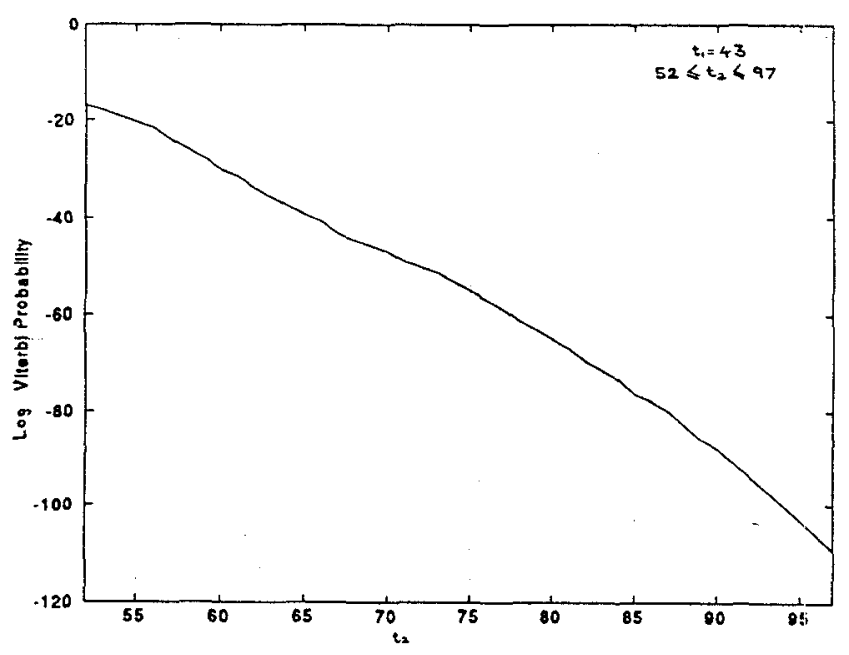

Figure 1. Variation of log Viterbi probability with time

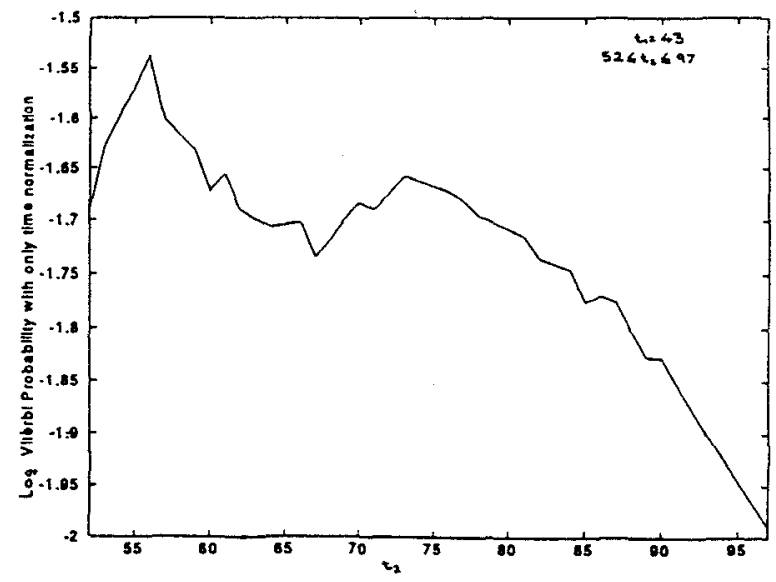

(a)

Time normalized log Viterbi probability vs. Time

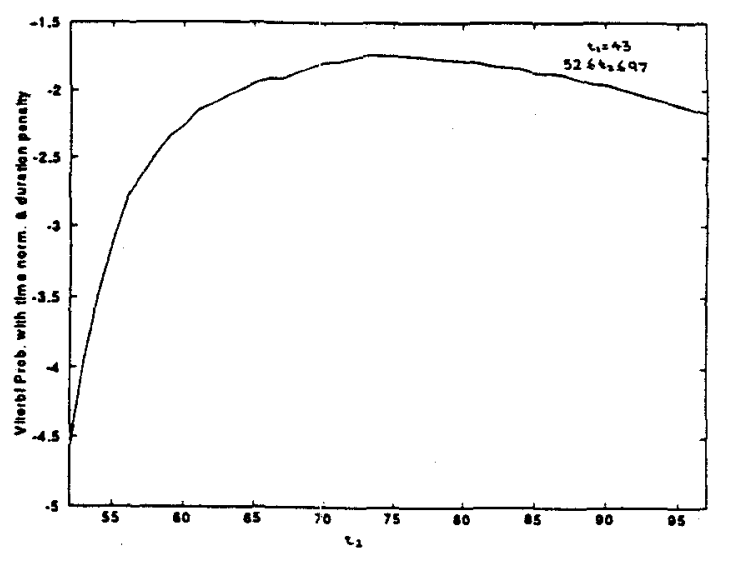

(b)

Time normalized log Vitérbi prob. and Duration penalty vs. Time

Figure 2. Scoring scheme 


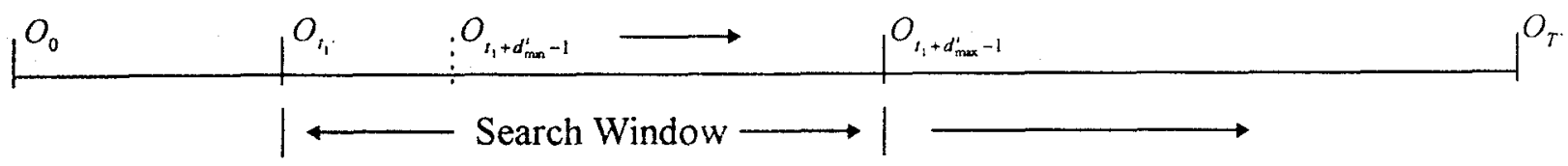

Figure 3. Search method.

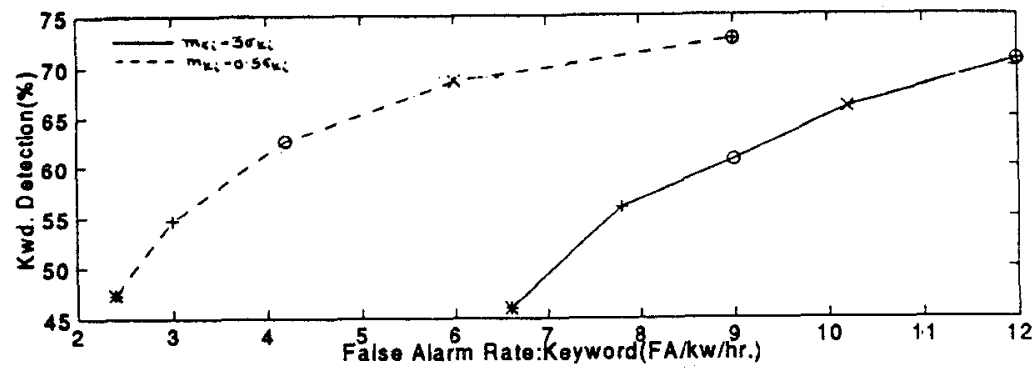

Figure 4. System performance as a function of thresholds for two values of $d_{i}^{\text {min }}$

Table 1. Results of No garbage model keyword spotting technique

\begin{tabular}{|c|c|c|c|}
\hline Data Set & No. of sentences & Detection Rate (\%) & $\begin{array}{c}\text { False Alarm Rate } \\
\text { ( FA/kw/hr. ) }\end{array}$ \\
\hline Train & 250 & 96.80 & 2.66 \\
\hline Test & 150 & 69.33 & 4.80 \\
\hline
\end{tabular}

Table 2. Duration Statistics

\begin{tabular}{|c|c|c|c|c|c|}
\hline Data Set & $\begin{array}{c}\text { No. of } \\
\text { sentences }\end{array}$ & $\begin{array}{c}\text { Mean error } \\
\text { in start pt. }\end{array}$ & $\begin{array}{c}\text { Mean error } \\
\text { in end pt. }\end{array}$ & $\begin{array}{c}\text { Error variance } \\
\text { in start pt. }\end{array}$ & $\begin{array}{c}\text { Error variance } \\
\text { in end pt. }\end{array}$ \\
\hline Train & 250 & 2 & 2 & 5.52 & 11.33 \\
\hline Test & 150 & 2 & 2 & 7.19 & 10.88 \\
\hline
\end{tabular}

Table 3. Recognition performance for test data for variable $d_{i}^{\text {rin }}$

\begin{tabular}{|c|c|c|}
\hline$d_{i}{ }^{\text {min }}$ & Recognition rate (\%) & $\begin{array}{c}\text { False Alarm Rate } \\
\text { Fa/Kw/Hr }\end{array}$ \\
\hline$m_{i}-0.25 \sigma_{i}$ & 65.33 & 4.20 \\
\hline$m_{i}-0.5 \sigma_{i}$ & 69.33 & 4.80 \\
\hline$m_{i}-0.75 \sigma_{i}$ & 68.00 & 4.80 \\
\hline$m_{i}-\sigma_{i}$ & 68.67 & 5.40 \\
\hline$m_{i}-2 \sigma_{i}$ & 68.00 & 9.00 \\
\hline$m_{i}-3 \sigma_{i}$ & 68.00 & 9.00 \\
\hline$m_{i}-4 \sigma_{i}$ & 68.00 & 9.00 \\
\hline$m_{i}-5 \sigma_{i}$ & 68.00 & 9.00 \\
\hline
\end{tabular}

